were found, with phenotypes QRNG and PPNG being more frequent.

Conclusion Although N. gonorrhoeae isolates with resistance to gentamicin were not observed, a high percentage of strains were resistant to other antimicrobial agents, particularly ciprofloxacin. This study suggests that gentamicin may be considered a future treatment option for gonorrhoea in Argentina. However, the high prevalence of isolates with MIC $8 \mu \mathrm{g} / \mathrm{ml}$ (intermediate susceptibility) suggests a continuous surveillance of gentamicin in our country.

\section{P3.86 COMPARATIVE EVALUATION OF DISK DIFFUSION AND AGAR DILUTION METHODS FOR GENTAMICIN SUSCEPTIBILITY TESTING OF NEISSERIA GONORRHOEAE}

${ }^{1} \mathrm{R}$ Gianecini, ${ }^{1} \mathrm{C}$ Oviedo, ${ }^{2} \mathrm{~L}$ Irazu, ${ }^{2} \mathrm{M}$ Rodríguez, ${ }^{3} \mathrm{G}$ ASSP-AR, ${ }^{1}$ Galarza P. ${ }^{1}$ Servicio de Enfermedades de Transmisión Sexual, Instituto Nacional de Enfermedades Infecciosas (INEI)ANLIS "Dr. Carlos G. Malbrán", Ciudad Autónoma de Buenos Aires, Argentina.; ${ }^{2}$ Departamento de Parasitología, Instituto Nacional de Enfermedades Infecciosas (INEI)ANLIS "Dr. Carlos G. Malbrán", Ciudad Autónoma de Buenos Aires, Argentina; ${ }^{3}$ Gonococcal Antimicrobial Susceptibility Surveillance Programme-Argentina (GASSP-AR)

\subsection{6/sextrans-2017-053264.32}

Introduction At present, gentamicin is a promising antibiotic for the treatment of multidrug resistant $N$. gonorrhoeae isolates. Therefore, the knowledge of N. gonorrhoeae susceptibility to gentamicin is required. The CLSI guidelines do not list breakpoints for gentamicin. However, MIC interpretive criteria have been proposed. Moreover, a recent report comparing the disk diffusion with Etest has established tentative gentamicin zone breakpoints for the CLSI method. The proposed breakpoints are $\geq 16 \mathrm{~mm}$ for susceptible, $13-15 \mathrm{~mm}$ for intermediate and $\leq 12 \mathrm{~mm}$ for resistant. The aim of this study was to compare the disk diffusion method with the agar dilution test, and to analyse the suitability and reliably of disk diffusion to monitor the susceptibility to gentamicin.

Methods We studied $237 \mathrm{n}$. gonorrhoeae isolates obtained in 2013 and 2015 from the GASSP-AR. The MIC determination and disk diffusion tests to gentamicin were performed according to CLSI, and tentative breakpoints previously reported were used. The 2008 WHO and ATCC 49226 reference strains were used as control. The inhibition diameters by disk diffusion were tested by correlation with the MIC value.

Results Gentamicin MICs ranged from 2 to $16 \mu \mathrm{g} / \mathrm{ml}$, and the MIC $_{50 / 90}$ were both $8 \mu \mathrm{g} / \mathrm{ml}$. The Pearson correlation between disk diffusion and agar dilution was $-0.67 \quad(\mathrm{p}<0.001)$. No very major or major discrepancies were detected with disk diffusion as compared to agar dilution. However, a high percentage of minor discrepancies (39.9\%) was observed. By adjusting the susceptible breakpoint for disk diffusion to $S \geq 17$ $\mathrm{mm}$, the minor discrepancies rate was reduced from $39.9 \%$ to $18.4 \%$.

Conclusion: N. gonorrhoeae isolates with resistance to gentamicin were not observed. The disk diffusion had good correlation when compared with the agar dilution method. Although a high percentage of minor discrepancies was observed, the error rate was reduced adjusting the breakpoint. Until it becomes standardised, the disk diffusion can be a screening method in clinical laboratories to detect the gentamicin susceptibility of N. gonorrhoeae.

\section{P3.87 ASYMPTOMATIC URETHRITIS PREVALENCE AND ASSOCIATED RISK FACTORS AMONG MALES LIVING WITH HIV-1}

Guilherme Almeida Rosa da Silva. UNIRIO, Rio De Janeiro - RJ, Brazil

\subsection{6/sextrans-2017-053264.322}

Introduction It is estimated that symptomatic carriers of $N$. gonorrhoeae and C. trachomatis have a relative risk of 4.8 fold and 3.6 fold, respectively, for the sexual acquisition of HIV. This type of evaluation for asymptomatic urethritis is necessary to reinforce strategies to combat HIV transmission.

Methods We enrolled a total of 115 male patients aged 18 years or older who have been diagnosed with an HIV infection and have no symptoms of urethritis or other sexually transmitted infections. Urine was collected and tested by realtime PCR for the detection of C. trachomatis and N. gonorrhoeae (Abbott RealTime CT/NG test ${ }^{\circledR}$ ). Simultaneous clinical data and blood collection was performed to obtain HIV viral load values and $\mathrm{CD} 4^{+} \mathrm{T}$ lymphocyte counts.

Results Four patients had asymptomatic carrier status of urethritis and were positive for C. trachomatis. The prevalence was $3.47 \%$. Patients who were positive for C. trachomatis urethritis had a lower mean age $(\mathrm{p}=0.015)$. After statistical analysis, we observe that age is associated with the time of use of ART $\left(\beta=\right.$ positive, $\left.\mathrm{R}^{2}=0.082, \mathrm{p}<0.01\right)$ and that the mean age of patients who do not use condoms is higher than those who use condoms in more than or equal to $50 \%$ of the relationships $(p=0.03)$. Likewise, the mean age in patients who use condoms in less than $50 \%$ of relationships is higher than that of patients who use condoms in more than or equal to $50 \%$ of relationships $(p=0.04)$. The status of single men is associated with sexual intercourse with other men or men and women and condom use in more than 50\% of relationships $(p<0.01)$. Uncircumcised men are associated with the highest plasma HIV viral load among patients with detectable HIV load when compared to circumcised men $(\mathrm{p}<0.01)$.

Conclusion We recommend that, in outpatient practice, the habit of inquiring about history of sexual behaviour to obtain more information about risks and associations with asymptomatic sexually transmitted infection, a routine physical examination and complementary tests for the agents should be discarded.

\section{P3.88 FREQUENCY OF SYPHILIS TESTING AMONG MEN WHO HAVE HAD RECTAL TESTS FOR CHLAMYDIA AND GONORRHOEA, UNITED STATES}

${ }^{1}$ Guoyu Tao, ${ }^{1}$ Thomas Peterman, ${ }^{2}$ Melinda B Nye, ${ }^{1}$ Thomas L Gift, ${ }^{2}$ Barbara A Body. ${ }^{1} C D C$, Atlanta, USA; ${ }^{2}$ LABCORP, Burlington, USA

\subsection{6/sextrans-2017-053264.323}

Introduction CDC recommends syphilis screening at least annually for sexually active MSM and screening every 3-6 months for MSM with risks such as multiple partners.

Methods In collaboration with a large U.S. commercial laboratory, we identified men aged $15-60$ years who had rectal chlamydia (CT) or gonorrhoea (GC) testing during September, 2013-Augest, 2015 as presumed MSM. We estimated: the frequency of testing (counting only tests $\geq 3$ months after a previous test); reactivity of nontreponemal tests (NTT) and treponemal tests (TT); and the association between CT/GC and the reactivity of syphilis NTT and TT. We also identified 